\title{
Correspondence
}

\section{Patient recruitment for clinical research}

To the Editor:

Dr. Wong's letter ${ }^{1}$ concerning patient recruitment for clinical research raises some interesting points concerning the time at which informed consent should be obtained and the implications of a "no exclusion" clause in a clinical research protocol.

We assume that the protocol was approved by the institutional ethics committee, although this is not stated in the letter. Were the three ocular block techniques for cataract surgery all commonly used? If so, they were not experimental techniques. If these assumptions are correct, the consent given by patients should have been only for collection and analysis of aggregate data for publication. Consent, either verbal or written, to have surgery performed under ocular block would have been obtained at the same time, and we are told that patients were made aware of the study by the surgeon. The informed consent for research, obtained shortly before anaesthesia and surgery, therefore refers to data collection concerning efficacy of the block rather than its performance.

We have never encountered a "no exclusion" clause in a study protocol and we are not sure what this could mean. The standard of care provided to patients must never be influenced by their decision to participate or not in a study protocol. Did all patients agree to participate and, if not, did they still have one of the three ocular blocks and their cataract surgery? The decision of the anaesthetist to proceed with the block in the patient described should have been based entirely on clinical criteria. We do not understand how postponement of surgery could have put the study at risk. The request of the patient, through his son, to proceed with the block and surgery was presumably because the patient wanted his vision restored and had nothing to do with the research protocol.

If there is a language barrier, the investigator, aided by an interpreter, must be satisfied that the patient understands what is entailed. The essential points to be covered include the reasons for the study, research techniques including randomization, anticipated benefits and consequences, foreseeable risks, maintenance of confidentiality of subjects, anticipated time commitment, and the right to withdraw from the study at any time and without penalty. ${ }^{2}$ If the investigator has any doubts about the patient's ability to comprehend, the patient must not be enrolled and this should have no consequences for the patient's medical care.

J.R. Maltby MB FRCPC

C.J. Eagle MD FRCPC

Department of Anaesthesia

Foothills Hospital and the University of Calgary

Calgary, Alberta

\section{REFERENCES}

1 Wong DHW. Patient recruitment for clinical research. Can J Anaesth 1993; 40: $287-8$.

2 Guidelines on Research Involving Human Subjects. Medical Research Council of Canada, 20th Floor, Jeanne Mance Building, Tunney's Pasture, Ottawa, Ontario, KIA 0W9, 1987; 22-3.

\section{$R E P L Y$}

I would like to thank Drs. Maltby and Eagle for their interest in the concerns I raised in regard to patient recruitment for clinical research.

The study protocol had been approved by both the university and hospital ethics committees on human experimentation. The study involved three ocular block techniques, one of which being an experimental approach. It involves, therefore, more than just data collection of established techniques. The nature and risks of the study were explained to the patients by the surgeon, who was a participating investigator, when they were seen at his office. On the day of surgery, these were explained to the patients again by the investigator-anaesthetist before written consent was signed.

The "no exclusion" term was used for the sake of brevity in my letter. The study involved day-care patients having cataract surgery at our Eye Care Centre (ECC). All patients were prescreened according to the criteria of the ECC with regard to their suitability for day-care surgery. The protocol stated that all patients who were accepted for ECC surgery would be suitable candidates for the study, and would not be excluded from recruitment because of age or medical problems, subject to patient consent. The "no exclusion" did not mean that all patients had to be in the study. The standard of care was not influenced by whether or not they participated, and this was made clear to the patients before seeking their consent. All patients gladly participated in the study. If the patient had refused to participate, a traditional technique (peribulbar block) would have been used, and he/she would not have been included in the study. It was the patient and his son who were keen on proceeding with the surgery and participating in the study, despite the assurance that withdrawing from the study would in no way compromise his care.

In regards to obtaining consent through an interpreter, all the points listed by Drs. Maltby and Eagle were explained ver- 